\title{
SPIN STRUCTURE FUNCTIONS IN CHIRAL QUARK SOLITON MODELS*
}

\author{
H. WEIGEL ${ }^{\dagger}$ \\ Institute for Theoretical Physics \\ Tübingen University \\ D-72076 Tübingen, Germany \\ E-mail: herbert.weigel@uni-tuebingen.de
}

\begin{abstract}
In this talk I review studies of hadron structure functions in bosonized chiral quark models. Such models require regularization and I show that the two-fold PauliVillars regularization scheme not only fully regularizes the effective action but also leads the scaling laws for structure functions. This scheme is consistent with other computations of the pion structure function in that model. For the nucleon structure functions the present approach serves to determine the regularization prescription for structure functions whose leading moments are not given by matrix elements of local operators. Some numerical results are presented for the spin structure functions and the role of strange quarks is addressed.
\end{abstract}

\section{Introduction}

In this talk I review the computation of hadron structure functions in the Nambu-Jona-Lasino (NJL) model ${ }^{1}$. This is a particularly simple model for quark interactions with the important feature that the quarks can be integrated out in favor of meson fields ${ }^{2}$. The resulting effective action for these mesons possesses soliton solutions ${ }^{3}$. According to the large $-N_{C}$ picture $^{4}$ of Quantum-Chromo-Dynamics (QCD) these solutions are interpreted as baryons. Quantization of the soliton then also yields baryon wave-functions in such meson models. The construction of hadron wave-functions is not possible in QCD. This represents the main obstacle for the computation of hadron properties from first principles. As the model adopts the symmetry properties of $\mathrm{QCD}$, the current operators in the model correspond to those of QCD. As a consequence matrix elements of the current operators as computed in the model are physical. In particular it is interesting to analyze the hadronic tensor that parameterizes the deep-inelastic-scattering (DIS)

\footnotetext{
*Based on plenary talk presented at the QCD-Workshop in Charlottesville, April 2002.

${ }^{\dagger}$ Heisenberg-Fellow
} 
and compare the model predictions with empirical data. This picture has led to interesting studies of hadron structure functions in bosonized chiral

quark models. In this talk I will present the results of refs. ${ }^{5,6,7,8}$. These studies build up a consistent approach by computing the hadronic tensor (or equivalently the forward virtual Compton amplitude) from the gauged meson action. For the nucleon structure functions similar studies have been reported in refs. ${ }^{9,10,11}$. There no attempt to compute the structure functions from the gauged action was made but rather the less convincing assumption was made that the model predictions for the constituent quark distribution can be identified with QCD quark distributions. However, I refer to those articles for a more expatiated presentation of numerical results.

This talk is organized as follows. In Section $2 \mathrm{I}$ introduce the NJL model as an effective meson theory and utilize pion properties to determine the model parameters. Section 3 describes the subtleties for extracting the structure functions that arise in this model from regularization. The pion structure function is considered as an example. In Section 4 I review the construction of baryon states in the soliton picture. The following Section sketches the computation of nucleon matrix elements of the hadronic tensor and the extraction of the structure functions in the Bjorken limit. Finally in Section 6 I present some numerical results for the spin structure functions $g_{1}$ and $g_{2}$ and compare them to experimental data by means of the transformation to the infinite momentum frame and subsequent DGLAP evolution. Section 7 serves as a short summary.

\section{The NJL Model for Chiral Dynamics}

The NJL model is a quark model with a chirally invariant four quark interaction. Semiclassical bosonization is achieved via introduction of effective meson fields for the quark bilinears in that interaction. Then the quark fields can be integrated out by functional methods. This yields an effective action for meson degrees of freedom,

$$
\mathcal{A}[S, P]=-i N_{C} \operatorname{Tr}_{\Lambda} \log \left[i \not \partial-\left(S+i \gamma_{5} P\right)\right]-\frac{1}{4 G} \int d^{4} x \operatorname{tr} \mathcal{V}(S, P) .
$$

Here $\mathcal{V}$ is a local potential respectively for the effective scalar and pseudoscalar fields $S$ and $P$ that are matrices in flavor space. In the NJL model it reads $\mathcal{V}=S^{2}+P^{2}+2 \hat{m}_{0} S$ with $\hat{m}_{0}$ being the current quark mass matrix. Since the interaction is mediated by flavor degrees of freedom, the number of colors is merely a multiplicative quantity. The functional trace (Tr) originates from integrating out the quarks and induces a non-local interaction 
for $S$ and $P$. For simplicity I will only consider the isospin limit for up (u) and down (d) quarks: $m_{0, u}=m_{0, d}=m_{0}$.

A major concern in regularizing the functional (1), as indicated by the cut-off $\Lambda$, is to maintain the chiral anomaly. This is achieved by splitting this functional into $\gamma_{5}$-even and odd pieces and only regulate the former,

$$
\begin{aligned}
\operatorname{Tr}_{\Lambda} \log \left[i \not \partial-\left(S+i \gamma_{5} P\right)\right]=- & i \frac{N_{C}}{2} \sum_{i=0}^{2} c_{i} \operatorname{Tr} \log \left[-\mathbf{D D}_{5}+\Lambda_{i}^{2}-i \epsilon\right] \\
& -i \frac{N_{C}}{2} \operatorname{Tr} \log \left[-\mathbf{D}\left(\mathbf{D}_{5}\right)^{-1}-i \epsilon\right]
\end{aligned}
$$

with

$$
i \mathbf{D}=i \not \partial-\left(S+i \gamma_{5} P\right) \quad \text { and } \quad i \mathbf{D}_{5}=-i \not \partial-\left(S-i \gamma_{5} P\right) \text {. }
$$

The double Pauli-Villars regularization renders the functional (1) finite with $c_{0}=1, \Lambda_{0}=0, \sum_{i=0}^{2} c_{i}=0$. The $\gamma_{5}$-odd piece is conditionally finite and not regularizing it properly reproduces the chiral anomaly. For sufficiently large $G$ one obtains the $\mathrm{VEV},\langle S\rangle \equiv m \mathbb{1}$ that parameterizes the dynamical chiral symmetry breaking, from the gap-equation,

$$
\frac{1}{2 G}\left(m-m_{0}\right)=-4 i N_{C} m \sum_{i=0}^{2} c_{i} \int \frac{d^{4} k}{(2 \pi)^{4}}\left[-k^{2}+m^{2}+\Lambda_{i}^{2}-i \epsilon\right]^{-1} .
$$

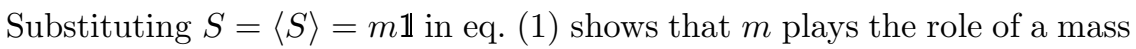
and is referred to as the constituent quark mass.

In the next step I utilize pion properties to fix the model parameters and introduce the pion field $\vec{\pi}$ via

$$
S+i P \gamma_{5}=m(U)^{\gamma_{5}}=m \exp \left(i \frac{g}{m} \gamma_{5} \vec{\pi} \cdot \vec{\tau}\right)
$$

Sandwiching the axial current between the vacuum and a single pion state yields the pion decay constant $f_{\pi}=93 \mathrm{MeV}$ in terms of the polarization function $\Pi\left(q^{2}, x\right)$,

$$
\begin{gathered}
f_{\pi}=4 N_{C} m g \int_{0}^{1} d x \Pi\left(m_{\pi}^{2}, x\right) \\
\Pi\left(q^{2}, x\right)=\sum_{i=0}^{2} c_{i} \frac{d^{4} k}{(2 \pi)^{4} i}\left[k^{2}+x(1-x) m_{\pi}^{2}-m^{2}-\Lambda_{i}^{2}+i \epsilon\right]^{-2}
\end{gathered}
$$

where $m_{\pi}=138 \mathrm{MeV}$ is the pion mass. The Yukawa coupling constant, $g$, is determined by the requirement that the pion propagator has unit residuum,

$$
\frac{1}{g^{2}}=4 N_{C} \frac{d}{d m_{\pi}^{2}} \int_{0}^{1} d x\left[m_{\pi}^{2} \Pi\left(m_{\pi}^{2}, x\right)\right]
$$


In the chiral limit $\left(m_{\pi}=0\right)$ this simplifies to $f_{\pi}=m / g$. Finally the current quark mass is fixed from the condition that the pole of the pion propagator is exactly at the pion mass,

$$
m_{0}=4 N_{C} m G m_{\pi}^{2} \int_{0}^{1} d x \Pi\left(m_{\pi}^{2}, x\right) .
$$

It is also worthwhile to mention that expanding eqs. (2) and (5) to linear and quadratic order in $\vec{\pi}$ and $v_{\mu}$, respectively yields the proper result for the anomalous decay $\pi^{0} \rightarrow \gamma \gamma$. This is the direct consequence of not regularizing the $\gamma_{5}$-odd piece.

Before discussing nucleons as solitons of the bosonized action (1) and the respective structure functions it will be illuminating to first consider DIS off pions.

\section{The Compton Tensor and Pion Structure Function}

DIS off hadrons is parameterized by the hadronic tensor $W^{\mu \nu}(q)$ where $q$ is the momentum transmitted from the photon to the hadron.

The tensor $W^{\mu \nu}(q)$ is obtained from the hadron matrix element of the current commutator by Fourier transformation and is parameterized in terms of form factors that multiply the allowed Lorentz structures. By pertinent projection of the hadron tensor these form factors can be extracted. Finally the structure functions are the leading twist contributions of the form factors. These contributions are obtained from computing $W^{\mu \nu}(q)$ in the Bjorken limit: $q^{2} \rightarrow-\infty$ with $x=-q^{2} / p \cdot q$ fixed.

An essential feature of bosonized quark models is that the derivative term in (1) is formally identical to that of a non-interacting quark model. Hence the current operator is given as $J^{\mu}=\bar{q} \mathcal{Q} \gamma^{\mu} q$, with $\mathcal{Q}$ a flavor matrix. Expectation values of currents are computed by introducing pertinent sources $v_{\mu}$ in eq $(2)$

$$
i \mathbf{D} \longrightarrow i \mathbf{D}+\mathcal{Q} \psi \quad \text { and } \quad i \mathbf{D}_{5} \longrightarrow i \mathbf{D}_{5}-\mathcal{Q} \psi
$$

and taking appropriate derivatives. In bosonized quark models it is convenient to start from the absorptive part of the forward virtual Compton amplitude $^{\mathrm{a}}$

$$
\begin{aligned}
T^{\mu \nu}(q) & =\int d^{4} \xi \mathrm{e}^{i q \cdot \xi}\left\langle p, s\left|T\left(J^{\mu}(\xi) J^{\nu}(0)\right)\right| p, s\right\rangle \\
W^{\mu \nu}(q) & =\frac{1}{2 \pi} \operatorname{lm}\left[T^{\mu \nu}(q)\right],
\end{aligned}
$$

\footnotetext{
aThe momentum of the hadron is called $p$ and its spin eventually $s$.
} 
because the time--ordered product is straightforwardly obtained from

$T\left(J^{\mu}(\xi) J^{\nu}(0)\right)=\left.\frac{\delta^{2}}{\delta v_{\mu}(\xi) \delta v_{\nu}(0)} \operatorname{Tr}_{\Lambda} \log \left[i \not \partial-\left(S+i \gamma_{5} P\right)+\mathcal{Q} \psi\right]\right|_{v_{\mu}=0}$,

as defined from eq. (2) with the substitution (9).

Pion-DIS is characterized by a single structure function, $F(x)$. For its computation the pion matrix element in the Compton amplitude (10) must be specified. For virtual pion-photon scattering it is obtained by expanding eqs. (2) and (5) to second order in both, $\vec{\pi}$ and $v_{\mu}$. Due to the separation into $\mathbf{D}$ and $\mathbf{D}_{5}$ this calculation differs considerably from the simple evaluation of the 'handbag' diagram. For example, isospin violating and dimension-five operators appear for the action (2). Fortunately all isospin violating pieces cancel yielding

$$
F(x)=\frac{5}{9}\left(4 N_{C} g^{2}\right) \frac{d}{d m_{\pi}^{2}}\left[m_{\pi}^{2} \Pi\left(m_{\pi}^{2}, x\right)\right], \quad 0 \leq x \leq 1 .
$$

The same result is obtained by formal treatment of the divergent handbag diagram and ad hoc regularization ${ }^{12}$. The cancellation of the isospin violating pieces is a feature of the Bjorken limit: insertions of the pion field on the propagator carrying the infinitely large photon momentum can be safely ignored. Furthermore this propagator can be taken to be the one for non-interacting massless fermions. This implies that also the Pauli-Villars cut-offs can be omitted for this propagator. That, in turn, leads to the desired scaling behavior of the structure function in this model and is a particular feature of the Pauli-Villars regularization. A priori it is not obvious for an arbitrary regularization scheme that terms of the form $q^{2} / \Lambda_{i}^{2}$ drop out in the Bjorken limit.

From eqs (7) and (12) it is obvious that $F(x)=5 / 9$ for $0 \leq x \leq 1$ in the chiral limit $\left(m_{\pi}=0\right)$. It must be noted that this refers to the structure function at the (low) energy scale of the model. To compare with empirical data, that are at a higher energy scale, the DGLAG program of perturbative QCD has to be applied to $F(x)$. Such studies show good agreement with the data ${ }^{13}$.

\section{The Nucleon as a Chiral Soliton}

Solitons are a non-perturbative and stationary configurations of the meson fields. To determine that configuration for the meson theory (1) I consider the hedgehog ansatz

$$
U_{\mathrm{H}}(\vec{r})=\exp (i \vec{\tau} \cdot \hat{r} F(r)) \quad \text { and } \quad\left(U_{\mathrm{H}}(\vec{r})\right)^{\gamma_{5}}=\exp \left(i \gamma_{5} \vec{\tau} \cdot \hat{r} F(r)\right)
$$


for the pion field (5). The corresponding single particle Dirac Hamiltonian reads

$$
h=\vec{\alpha} \cdot \vec{p}+\beta m\left[\cos F+i \gamma_{5} \vec{\tau} \cdot \hat{r} \sin F\right] .
$$

Evaluating the action functional (1) in the eigenbasis of $h$ gives the energy functional ${ }^{14}$ in terms of the eigenvalues, $\epsilon_{\alpha}$,

$$
\begin{gathered}
E[F]=\frac{N_{C}}{2}\left(1-\operatorname{sign}\left(\epsilon_{\mathrm{V}}\right)\right) \epsilon_{\mathrm{V}}-\frac{N_{C}}{2} \sum_{\alpha} \sum_{i=0}^{2} c_{i}\left\{\sqrt{\epsilon_{\alpha}^{2}+\Lambda_{i}^{2}}-\sqrt{\epsilon_{\alpha}^{(0) 2}+\Lambda_{i}^{2}}\right\} \\
+m_{\pi}^{2} f_{\pi}^{2} \int d^{3} r(1-\cos F)
\end{gathered}
$$

for a baryon number one configuration. Here $\mathrm{V}$ denotes the unique quark level that is strongly bound by the soliton and $\epsilon_{\alpha}^{(0)}$ are the eigenvalues when the soliton is absent. The soliton profile $F(r)$ is then obtained from extremizing $E$ self-consistently ${ }^{3}$.

States possessing good spin and isospin quantum numbers are generated by taking the zero-modes to be time dependent ${ }^{15}$

$$
U(\vec{r}, t)=A(t) U_{\mathrm{H}}(\vec{r}) A^{\dagger}(t),
$$

which introduces the collective coordinates $A(t) \in S U(2)$. The action functional is expanded ${ }^{16}$ up to quadratic order in the angular velocities

$$
i \vec{\tau} \cdot \vec{\Omega}=2 A^{\dagger}(t) \dot{A}(t) .
$$

The coefficient of the quadratic ${ }^{\mathrm{b}}$ term defines the moment of inertia ${ }^{\mathrm{c}}, \alpha^{2}[F]$. Nucleon states $|N\rangle$ are obtained by canonical quantization of the collective coordinates, $A(t)$. This is analogous to quantizing a rigid rotator. The eigenfunctions are the well-known Wigner $D$-functions

$$
\langle A \mid N\rangle=\frac{1}{2 \pi} D_{I_{3},-J_{3}}^{1 / 2}(A),
$$

with $I_{3}$ and $J_{3}$ being respectively the isospin and spin projection quantum numbers of the nucleon. This then allows to compute matrix elements of operators in the space of the collective coordinates ${ }^{15}$ :

$$
\left\langle N\left|\frac{1}{2} \operatorname{tr}\left(\tau_{a} A^{\dagger} \tau_{b} A\right)\right| N\right\rangle=-\frac{4}{3}\left\langle N\left|I_{a} J_{b}\right| N\right\rangle \quad \text { and } \quad \vec{\Omega}=-\vec{J} / \alpha^{2}[F] .
$$

\footnotetext{
${ }^{\mathrm{b}} \mathrm{A}$ liner term does not arise due to isospin symmetry. ${ }^{\mathrm{c}}$ Functional integrals are evaluated using the eigenfunctions $\phi_{\alpha}$ of the Dirac Hamiltonian (13) in the background of the chiral angle $F(r)$. Thus all quantities - like the moment of inertia - turn into functionals of $F(r)$.
} 
For later use I note that the valence quark wave-function receives a first order perturbation

$$
\Psi_{\mathrm{V}}(\vec{r}, t)=\mathrm{e}^{-i \epsilon_{\mathrm{V}} t} A(t)\left\{\phi_{\mathrm{V}}(\vec{r})+\frac{1}{2} \sum_{\mu \neq \mathrm{V}} \phi_{\mu}(\vec{r}) \frac{\langle\mu|\vec{\tau} \cdot \vec{\Omega}| \mathrm{V}\rangle}{\epsilon_{\mathrm{V}}-\epsilon_{\mu}}\right\} .
$$

The moment of inertia, $\alpha^{2}[F]$ is order $N_{C}$, thus, upon quantization (18), this rotational correction is subleading in $1 / N_{C}$.

Also, later in this talk I will generalize the soliton approach to the case of three flavors. The quantization of the soliton proceeds along the same line by taking $A(t) \in S U(3)$. However, there is one essential difference: $S U(3)$ is only an approximate symmetry because the current mass of the strange quark differs substantially from that of the up and down quarks. This will be reflected in the Hamiltonian for the collective coordinates $A(t)$ by an explicit flavor symmetry breaking contribution. Nevertheless this Hamiltonian can still be diagonalized exactly by numerical means ${ }^{17}$. As a result the eigenfunctions are not simple Wigner $D$-functions of specific $S U(3)$ representations but rather linear combinations thereof. These linear combinations reflect the fact that spin- $\frac{1}{2}$ baryons are no longer pure octet states but acquire admixture of higher dimensional $S U(3)$ representations. For example the nucleon becomes

$|N\rangle=|N, \mathbf{8}\rangle+c_{1}[F] \frac{m_{s}-m}{m_{s}+m}|N, \overline{\mathbf{1 0}}\rangle+c_{2}[F] \frac{m_{s}-m}{m_{s}+m}|N, \mathbf{2 7}\rangle+\ldots$

where the constants of proportionality, $c_{i}$ are computed from the selfconsistent soliton. The exact wave-functions will be employed when studying nucleon properties in the three flavor model. For further details on quantizing the soliton in flavor $S U(3)$ I refer to the reviews ${ }^{18}$.

\section{Nucleon Structure Functions}

DIS off nucleons is described by four structure functions: $F_{1}(x)$ and $F_{2}(x)$ are insensitive to the nucleon spin. Those associated with the components of the hadronic tensor that contain the nucleon spin are $g_{1}(x)$ and $g_{2}(x)$.

As argued in section 3 , the quark propagator with the infinite photon momentum should be taken to be the one for free and massless fermions. Thus, it is sufficient to differentiate (Here $\mathbf{D}$ and $\mathbf{D}_{5}$ are those of eq (3), i.e. with $v_{\mu}=0$.)

$$
\begin{aligned}
& \frac{N_{C}}{4 i} \sum_{i=0}^{2} c_{i} \operatorname{Tr}\left\{\left(-\mathbf{D D}_{5}+\Lambda_{i}^{2}\right)^{-1}\left[\mathcal{Q}^{2} \psi(\not \partial)^{-1} \psi \mathbf{D}_{5}-\mathbf{D}\left(\psi(\not \partial)^{-1} \psi\right)_{5} \mathcal{Q}^{2}\right]\right\} \\
& \quad+\frac{N_{C}}{4 i} \operatorname{Tr}\left\{\left(-\mathbf{D D}_{5}\right)^{-1}\left[\mathcal{Q}^{2} \psi(\not \partial)^{-1} \psi \mathbf{D}_{5}+\mathbf{D}\left(\psi(\not \partial)^{-1} \psi\right)_{5} \mathcal{Q}^{2}\right]\right\},
\end{aligned}
$$


with respect to the photon field $v_{\mu}$. I have introduced the $(\ldots)_{5}$ description

$$
\gamma_{\mu} \gamma_{\rho} \gamma_{\nu}=S_{\mu \rho \nu \sigma} \gamma^{\sigma}-i \epsilon_{\mu \rho \nu \sigma} \gamma^{\sigma} \gamma^{5}, \quad\left(\gamma_{\mu} \gamma_{\rho} \gamma_{\nu}\right)_{5}=S_{\mu \rho \nu \sigma} \gamma^{\sigma}+i \epsilon_{\mu \rho \nu \sigma} \gamma^{\sigma} \gamma^{5}
$$

to account for the unconventional appearance of axial sources in $\mathbf{D}_{5}$, cf. ref. ${ }^{8}$. Substituting (15) for the meson fields that are contained in $\mathbf{D}$ and $\mathbf{D}_{5}$, computing the functional trace up to subleading order in $1 / N_{C}$ using a basis of quark states obtained from the Dirac Hamiltonian (13), yields analytical results for the structure functions. I refer to ref. ${ }^{8}$ for detailed formulae for other structure functions and the verification of the sum rules that relate integrals over the structure functions to static nucleon properties. As an example I restrain myself to list the contribution to $g_{1}(x)$ which is leading order in $1 / N_{C}$ :

$$
\begin{array}{r}
g_{1}(x)=\frac{M_{N} N_{C}}{36 i}\left\langle N\left|I_{3}\right| N\right\rangle \int \frac{d \omega}{2 \pi} \sum_{\alpha} \int d^{3} \xi \int \frac{d \lambda}{2 \pi} \mathrm{e}^{i M_{N} x \lambda} \\
\times\left(\sum_{i=0}^{2} \frac{c_{i}\left(\omega+\epsilon_{\alpha}\right)}{\omega^{2}-\epsilon_{\alpha}^{2}-\Lambda_{i}^{2}+i \epsilon}\right)_{\mathrm{P}}\left[\phi_{\alpha}^{\dagger}(\vec{\xi}) \tau_{3}\left(1-\alpha_{3}\right) \gamma_{5} \phi_{\alpha}\left(\vec{\xi}+\lambda \hat{e}_{3}\right) \mathrm{e}^{-i \omega \lambda}\right. \\
\left.\quad+\phi_{\alpha}^{\dagger}(\vec{\xi}) \tau_{3}\left(1-\alpha_{3}\right) \gamma_{5} \phi_{\alpha}\left(\vec{\xi}-\lambda \hat{e}_{3}\right) \mathrm{e}^{i \omega \lambda}\right],
\end{array}
$$

where the subscript $(P)$ indicates the pole term.

Before discussing numerical results I would like to mention the unexpected result that the structure function entering the Gottfried sum rule is related to the $\gamma_{5}$-odd piece of the action and hence does not undergo regularization. This is surprising because in the parton model this structure function differs from the one associated with the Adler sum rule only by the sign of the anti-quark distribution. The latter structure function, however, gets regularized in the present model, in agreement with the quantization rules for the collective coordinates.

\section{Numerical Results for Spin Structure Functions}

Unfortunately numerical results for the full structure functions in the double Pauli-Villars regularization scheme, i.e. including the properly regularized vacuum piece are not yet available. However, in the Pauli-Villars regularization the axial charges are saturated to $95 \%$ or more by their valence quark (19) contributions once the self-consistent soliton is substituted. This provides sufficient justification to consider the valence quark contribution to the polarized structure functions as a reliable approximation since e.g. the zeroth moment of the leading structure function $g_{1}$ is nothing but the axial current matrix element. This valence quark level is that of the chiral 
soliton model and its contributions to the structure functions should not be confused with valence quark distributions in parton models. In general, it should be stressed that the present model calculation yields structure functions, i.e. quantities that parameterize the hadronic tensor, but not (anti)-quark distributions. The latter would require the identification of model degrees of freedom with those in QCD. However, here only the symmetries (namely the chiral symmetry) and thus the current operators in the hadronic tensor are identified.

As in the case for the pion, the model calculation yields the nucleon structure function at a low energy scale. In addition it must be noted that the soliton is a localized object and thus not a representation of the Poincaré group. As a result the computation of structure functions is not frame-independent. It is appropriate to choose the infinite momentum frame (IMF) not only because it makes contact with the parton model but also because it is that frame in which the support of the structure functions is limited to the physical regime $0 \leq x \leq 1$. Choosing the IMF amounts to the transformation ${ }^{19,20}$

$$
f_{\mathrm{IMF}}(x)=\frac{1}{1-x} f_{\mathrm{RF}}(-\ln (1-x))
$$

where $f_{\mathrm{RF}}(x)$ denotes the structure function as computed in the nucleon rest frame. So the program is two-stage, first the transformation of the model structure function to the IMF according to eq (22) and subsequently the DGLAP evolution program ${ }^{21}$ to incorporate the $\ln \left|q^{2}\right|$ corrections. In the current context it is appropriate to restrain oneself to the leading order (in $\alpha_{s}$ ) in the evolution program because higher orders require to identify quark and antiquark distributions in the parton models sense. In the present model calculation this is not possible without further assumptions ${ }^{\mathrm{d}}$. The low energy scale, $Q_{0}^{2}=0.4 \mathrm{GeV}^{2}$, at which the model is assumed to approximate QCD has been estimated in ref. ${ }^{5}$ from a best fit to the experimental data of the unpolarized structure function, $F_{2}(x)$. The same boundary value is taken to evolve the model prediction for polarized structure function, $g_{1}(x)$, in the IMF $(22)$ to the scale $Q^{2}$ of several $\mathrm{GeV}^{2}$ at which the experimental data are available. For the structure function $g_{2}(x)$ the situation is a bit more complicated. First the twist-2 piece must be separated according to ${ }^{22}$

$$
g_{2}^{W W}(x)=-g_{1}(x)+\int_{x}^{1} \frac{d y}{y} g_{1}(y)
$$

\footnotetext{
${ }^{\mathrm{d}}$ We assume, however, that the gluon distribution is zero at the model scale.
} 

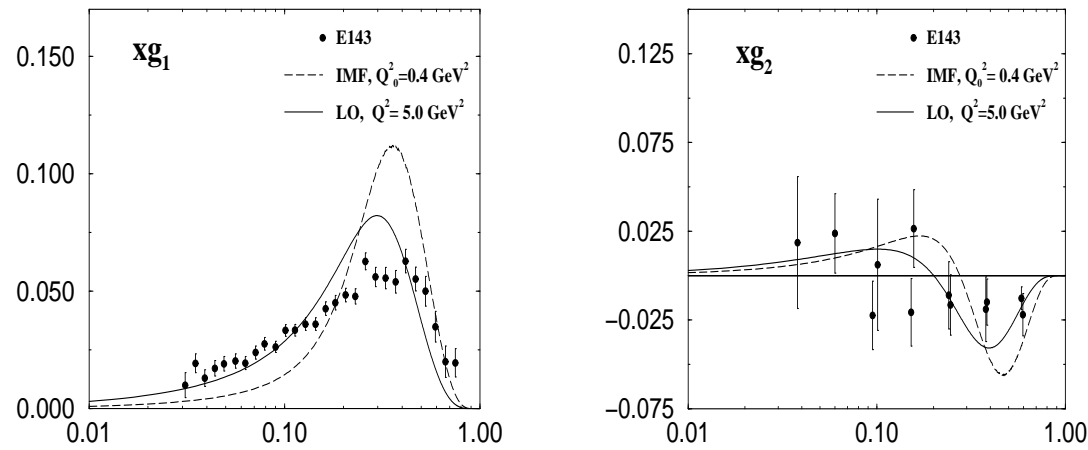

Figure 1. Model predictions for the polarized proton structure functions ${ }^{\mathbf{X}} x g_{1}$ (left panel) and $x g_{2}$ (right panel). The curves labeled 'RF' denote the results as obtained from the valence quark contribution to (20). These undergo a projection to the infinite momentum frame 'IMF' (22) and a leading order 'LO' DGLAP evolution ${ }^{21}$. Data are from SLAC-E143 ${ }^{24}$.

and evolved analogously to $g_{1}(x)$ (which also is twist-2). The remainder, $g_{2}(x)-g_{2}^{W W}(x)$ is twist -3 and is evolved according to the large $-N_{C}$ scheme of ref. ${ }^{23}$. Finally, the two pieces are again put together at the end-point of the evolution, $Q^{2}$. In figure 1 I compare the model predictions for the linearly independent polarized structure functions of the proton to experimental data ${ }^{24}$. In figure 2 I compare the model predictions for both the proton and the neutron (in form of the deuteron) not only to the recently accumulated data but also to other model predictions. Surprisingly the twist-2 truncation, i.e. eq (23) with the data for $g_{1}(x)$ at the right hand side gives the most accurate description of the data. However, also the chiral soliton model predictions reproduce the data well. Bag model predictions have a less pronounced structure.

As mentioned in section 4, the chiral soliton model can be generalized to three flavors. Appropriate projection gives a prediction for the strangeness contribution to structure functions ${ }^{7}$. In figure 3 this contribution is shown for the spin structure function $g_{1}(x)$. The interesting feature is that $g_{1}^{(s)}(x)$ has both positive and negative pieces. This is a nice example showing that a small $\Delta s=\int_{0}^{1} d x g_{1}^{(s)}(x)$ (strange quark contribution to the nucleon spin) does not imply that strangeness structure function itself is small.

\section{Conclusions}

I have presented studies of the nucleon spin structure functions in chiral soliton models. For this purpose I considered the bosonized NJL model as a simplified model for the quark flavor dynamics. Although the bosonized version is an effective meson theory, it has the interesting feature that the 


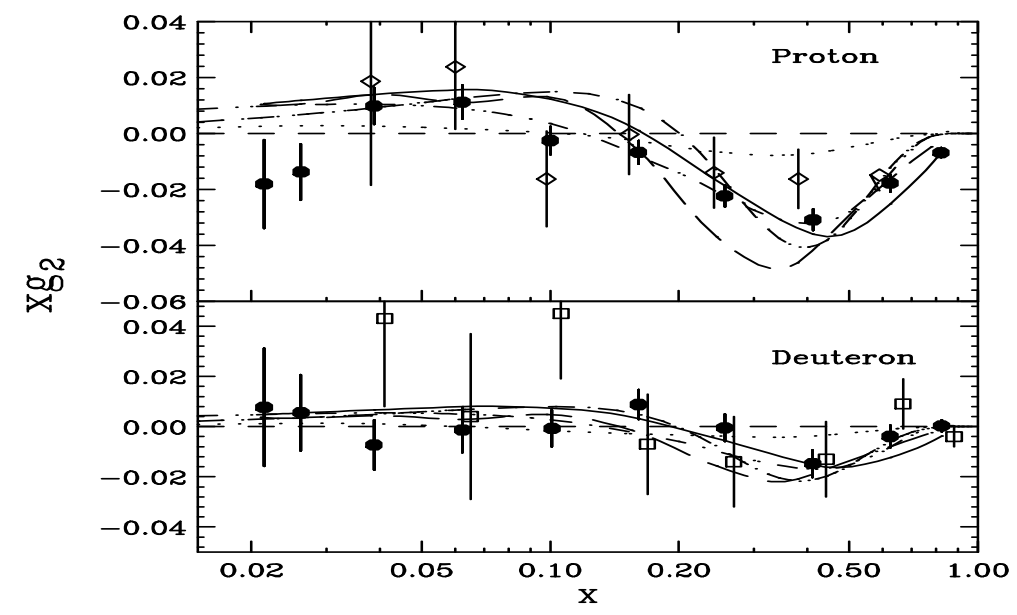

Figure 2. Model predictions for the polarized proton structure functions $x g_{2}$ for proton and neutron (deuteron) and comparison with data from $\mathrm{E} 143^{24}$ (open diamond) and $\mathrm{E} 155^{25}$ (open square) and their combination (solid circle). The full line is the twist-2 truncation (23) of data for $g_{1}(x)$. Dashed-dotted ${ }^{26}$ and dotted ${ }^{27}$ lines are bag model calculations, the short dashed lines represent the present chiral soliton model ${ }^{6}$ and long dashed line that of ref. ${ }^{11}$. (This is a slightly modified figure from ref. ${ }^{25}$.)

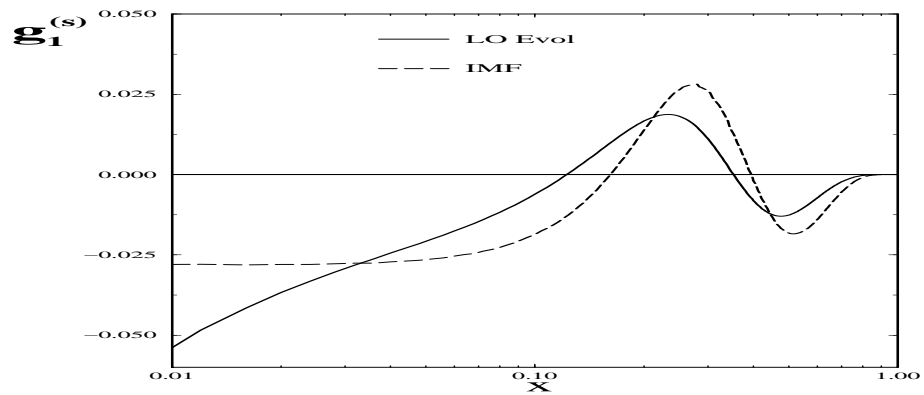

Figure 3. The strange quark contribution to the nucleon structure function $g_{1}(x)$ in the infinite momentum frame (IMF) and in leading order (LO) evolution from the model scale $Q_{0}^{2}=0.4 \mathrm{GeV}^{2}$ to $Q^{2}=3 \mathrm{GeV}^{2}$.

quark degrees of freedom can be traced. This is very helpful for considering structure functions. It turned out that additional correlations are introduced due to the unavoidable regularization which is imposed in a way to respect the chiral anomaly. Hence a consistent extraction of the nucleon structure functions from the Compton amplitude in the Bjorken limit leads to expressions that are quite different from those obtained by an ad hoc regularization of quark distributions in the same model. I also showed that within a reliable approximation the numerical results for the spin dependent 
structure functions agree reasonably well with the empirical data.

I would like to thank the organizers for this worthwhile workshop. The contributions of my colleagues L. Gamberg, H. Reinhardt, E. Ruiz Arriola and O. Schröder to this work are gratefully acknowledged. This work has been supported by the Deutsche Forschungsgemeinschaft under contract We 1254/3-2.

\section{References}

1. Y. Nambu and G. Jona-Lasinio, Phys. Rev. 122 (1961) 345; 124 (1961) 246.

2. D. Ebert and H. Reinhardt, Nucl. Phys. B271 (1986) 188.

3. R. Alkofer, H. Reinhardt and H. Weigel, Phys. Rept. 265 (1996) 139; C. V. Christov et al., Prog. Part. Nucl. Phys. 37 (1996) 91.

4. G. t' Hooft, Nucl. Phys. B72 (1974) 461; B75 (1975) 461. E. Witten, Nucl. Phys. B160 (1979) 57.

5. H. Weigel, L. Gamberg and H. Reinhardt, Mod. Phys. Lett. A11 (1996) 3021; Phys. Lett. B399 (1997) 287; L. Gamberg, H. Reinhardt and H. Weigel, Phys. Rev. D58 (1998) 054014; H. Weigel, Nucl. Phys. A670 (2000) 92.

6. H. Weigel, L. Gamberg and H. Reinhardt, Phys. Rev. D55 (1997) 6910.

7. O. Schröder, H. Reinhardt and H. Weigel, Phys. Lett. B439 (1998) 398.

8. H. Weigel, E. Ruiz Arriola and L. Gamberg, Nucl. Phys. B560 (1999) 383.

9. D. I. Diakonov et al., Nucl. Phys. B480 (1996) 341, Phys. Rev. D56 (1997) 4069; B. Dressler, K. Goeke, M. V. Polyakov and C. Weiss, Eur. Phys. J. C14 (2000) 147.

10. M. Wakamatsu and T. Kubota, Phys. Rev. D57 (1998) 5755; Phys. Rev. D60 (1999) 034020.

11. M. Wakamatsu, Phys. Lett. B 487 (2000) 118.

12. T. Frederico and G. A. Miller, Phys. Rev. D50 (1994) 210.

13. R. M. Davidson and E. Ruiz Arriola, arXiv:hep-ph/0110291.

14. F. Döring et al., Nucl. Phys. A536 (1992) 548.

15. G. S. Adkins, C. R. Nappi, and E. Witten, Nucl. Phys. B228 (1983) 552.

16. H. Reinhardt, Nucl. Phys. A503 (1989) 825.

17. H. Yabu and K. Ando, Nucl. Phys. B301 (1988) 601.

18. H. Weigel, Int. J. Mod. Phys. A11 (1996) 2419; J. Schechter and H. Weigel, arXiv:hep-ph/9907554.

19. R. L. Jaffe, Annals Phys. 132 (1981) 32.

20. L. Gamberg, H. Reinhardt and H. Weigel, Int. J. Mod. Phys. A13 (1998) 5519.

21. G. Altarelli, P. Nason and G. Ridolfi, Phys. Lett. B320 (1994) 152; B325 (1994) $538(\mathrm{E})$.

22. S. Wandzura and F. Wilczek, Phys. Lett. B 72 (1977) 195

23. A. Ali, V. M. Braun and G. Hiller, Phys. Lett. B266 (1991) 117

24. K. Abe et al., Phys. Rev. D58 (1998) 112003.

25. P. L. Anthony et al., arXiv:hep-ex/0204028.

26. M. Stratmann, Z. Phys. C 60 (1993) 763.

27. X. Song, Phys. Rev. D 54 (1996) 1955. 